\title{
Martingale convergence theorem for a conditional intuitionistic fuzzy mean value
}

\author{
Katarína Čunderlíková \\ Mathematical Institute, Slovak Academy of Sciences \\ Štefánikova 49, 81473 Bratislava, Slovakia \\ e-mail: cunderlikova. lendelova@gmail.com
}

Received: 5 January 2021

Accepted: 8 June 2021

\begin{abstract}
The aim of this contribution is to show a representation of a conditional intuitionistic fuzzy mean value of intuitionistic fuzzy observables by a conditional mean value of random variables. We formulate a martingale convergence theorem for a conditional intuitionistic fuzzy mean value, too.
\end{abstract}

Keywords: Intuitionistic fuzzy observable, Intuitionistic fuzzy state, Product, Conditional intuitionistic fuzzy mean value, Martingale convergence theorem.

2020 Mathematics Subject Classification: 03B52, 60A86, 60A10, 60 G48.

\section{Introduction}

In [4], we have defined a conditional intuitionistic fuzzy mean value $\mathbf{E}(y \mid x)=f$ as a Borel measurable function such that

$$
\mathbf{E}\left(y_{x(B)}\right)=\int_{B} \mathbf{E}(y \mid x) d \mathbf{m}_{x}
$$

for each $B \in \mathcal{B}(R)$, where $x, y: \mathcal{B}(R) \rightarrow R$ are the intuitionistic fuzzy observables, $y$ is integrable, i.e., the integral $\int_{R} t d \mathbf{m}_{y}(t)$ exists, $\mathbf{m}: \mathcal{F} \rightarrow[0,1]$ is the intuitionistic fuzzy state and an intuitionistic fuzzy observable $y_{x(B)}: \mathcal{B}(R) \rightarrow R$ is given by 


$$
y_{x(B)}(D)= \begin{cases}\left(0_{\Omega}, 1_{\Omega}\right), & \text { if } D=\emptyset \\ y(D \backslash\{0\}) \cdot x(B), & \text { if } D \neq R, D \in \mathcal{B}(R) \\ \left(1_{\Omega}, 0_{\Omega}\right), & \text { if } D=R .\end{cases}
$$

Generally, such a conditional mean value has many applications in regression analysis and in financial mathematics and insurance, so it is interesting to study convergence theorems. In the classical probability theory there is the martingale convergence theorem for random variables using conditional mean value (see [10]). In this paper, we try to formulate modification of a martingale convergence theorem for intuitionistic fuzzy observables using a conditional intuitionistic fuzzy mean value. We show the relation between a conditional intuitionistic fuzzy mean value of intuitionistic fuzzy observables and a conditional mean value of random variables, too.

The paper is organized as follows: Section 2 includes the basic notions from intuitionistic fuzzy probability theory such as intuitionistic fuzzy event, intuitionistic fuzzy state, intuitionistic fuzzy observable, intuitionistic fuzzy mean value and a joint intuitionistic fuzzy observable. In Section 3, we show a definition of conditional intuitionistic fuzzy mean value and the notions connected with it. In Section 4, we present the main result of the paper. First, we show a connection between a conditional intuitionistic fuzzy mean value of intuitionistic fuzzy observables and a conditional mean value of random variables. Then we formulate the martingale convergence theorem for a conditional intuitionistic fuzzy mean value of intuitionistic fuzzy observables. The last section contains concluding remarks.

We note that in the whole text we use a notation IF as an abbreviation for intuitionistic fuzzy.

\section{Elementary notions of the intuitionistic fuzzy probability theory}

The IF set was introduced by K. T. Atanassov in [1]. The IF set is a generalization of Zadeh's fuzzy set introduced in [11,12] given by $(f, 1-f)$, where $f$ is a fuzzy set. In this section we recall the definitions of elemetary notions connected with IF-probability theory.

Definition 2.1 ( $[2,3,9])$. Let $\Omega$ be a nonempty set. An IF-set $\mathbf{A}$ on $\Omega$ is a pair $\left(\mu_{A}, \nu_{A}\right)$ of mappings $\mu_{A}, \nu_{A}: \Omega \rightarrow[0,1]$ such that $\mu_{A}+\nu_{A} \leq 1_{\Omega}$.

Definition 2.2 ([2,3,9]). Start with a measurable space $(\Omega, \mathcal{S})$. Hence $\mathcal{S}$ is a $\sigma$-algebra of subsets of $\Omega$. An IF-event is called an IF-set $\mathbf{A}=\left(\mu_{A}, \nu_{A}\right)$ such that $\mu_{A}, \nu_{A}: \Omega \rightarrow[0,1]$ are $\mathcal{S}$-measurable.

The family of all IF-events on $(\Omega, \mathcal{S})$ will be denoted by $\mathcal{F}$, the mapping $\mu_{A}: \Omega \longrightarrow[0,1]$ will be called the membership function, the mapping $\nu_{A}: \Omega \longrightarrow[0,1]$ will be called the nonmembership function. 
If $\mathbf{A}=\left(\mu_{A}, \nu_{A}\right) \in \mathcal{F}, \mathbf{B}=\left(\mu_{B}, \nu_{B}\right) \in \mathcal{F}$, then we define the Lukasiewicz binary operations $\oplus, \odot$ on $\mathcal{F}$ by

$$
\begin{aligned}
& \left.\mathbf{A} \oplus \mathbf{B}=\left(\left(\mu_{A}+\mu_{B}\right) \wedge 1_{\Omega},\left(\nu_{A}+\nu_{B}-1_{\Omega}\right) \vee 0_{\Omega}\right)\right), \\
& \left.\mathbf{A} \odot \mathbf{B}=\left(\left(\mu_{A}+\mu_{B}-1_{\Omega}\right) \vee 0_{\Omega},\left(\nu_{A}+\nu_{B}\right) \wedge 1_{\Omega}\right)\right)
\end{aligned}
$$

and the partial ordering is given by

$$
\mathbf{A} \leq \mathbf{B} \Longleftrightarrow \mu_{A} \leq \mu_{B}, \nu_{A} \geq \nu_{B}
$$

In the IF-probability theory ( [8]) instead of the notion of probability we use the notion of state.

Definition 2.3 ([8]). Let $\mathcal{F}$ be the family of all IF-events in $\Omega$. A mapping $\mathbf{m}: \mathcal{F} \rightarrow[0,1]$ is called an IF-state, if the following conditions are satisfied:

(i) $\mathbf{m}\left(\left(1_{\Omega}, 0_{\Omega}\right)\right)=1, \mathbf{m}\left(\left(0_{\Omega}, 1_{\Omega}\right)\right)=0$;

(ii) if $\mathbf{A} \odot \mathbf{B}=\left(0_{\Omega}, 1_{\Omega}\right)$ and $\mathbf{A}, \mathbf{B} \in \mathcal{F}$, then $\mathbf{m}(\mathbf{A} \oplus \mathbf{B})=\mathbf{m}(\mathbf{A})+\mathbf{m}(\mathbf{B})$;

(iii) if $\mathbf{A}_{n} \nearrow \mathbf{A}\left(\right.$ i.e. $\left.\mu_{A_{n}} \nearrow \mu_{A}, \nu_{A_{n}} \searrow \nu_{A}\right)$, then $\mathbf{m}\left(\mathbf{A}_{n}\right) \nearrow \mathbf{m}(\mathbf{A})$.

The third basic notion in the probability theory is the notion of an observable. Let $\mathcal{J}$ be the family of all intervals in $R$ of the form

$$
[a, b)=\{x \in R: a \leq x<b\} .
$$

Then the $\sigma$-algebra $\sigma(\mathcal{J})$ is denoted $\mathcal{B}(R)$ and it is called the $\sigma$-algebra of Borel sets. Its elements are called Borel sets.

Definition 2.4 ([8]). By an IF-observable on $\mathcal{F}$ we understand each mapping $x: \mathcal{B}(R) \rightarrow \mathcal{F}$ satisfying the following conditions:

(i) $x(R)=\left(1_{\Omega}, 0_{\Omega}\right), x(\emptyset)=\left(0_{\Omega}, 1_{\Omega}\right)$;

(ii) if $A \cap B=\emptyset$, then $x(A) \odot x(B)=\left(0_{\Omega}, 1_{\Omega}\right)$ and $x(A \cup B)=x(A) \oplus x(B)$;

(iii) if $A_{n} \nearrow A$, then $x\left(A_{n}\right) \nearrow x(A)$.

If we denote $x(A)=\left(x^{b}(A), 1_{\Omega}-x^{\sharp}(A)\right)$ for each $A \in \mathcal{B}(R)$, then $x^{b}, x^{\sharp}: \mathcal{B}(R) \rightarrow \mathcal{T}$ are observables, where $\mathcal{T}=\{f: \Omega \rightarrow[0,1] ; f$ is $\mathcal{S}-$ measurable $\}$.

Similarly, as in the classical case, the following theorem can be proved $([7,8])$.

Theorem 2.5 ([7]). Let $x: \mathcal{B}(R) \longrightarrow \mathcal{F}$ be an IF-observable, $\mathbf{m}: \mathcal{F} \longrightarrow[0,1]$ be an IF-state. Define the mapping $\mathbf{m}_{x}: \mathcal{B}(R) \longrightarrow[0,1]$ by the formula

$$
\mathbf{m}_{x}(C)=\mathbf{m}(x(C)) .
$$

Then $\mathbf{m}_{x}: \mathcal{B}(R) \longrightarrow[0,1]$ is a probability measure. 
In [6] we introduced the notion of product operation on the family of IF-events $\mathcal{F}$ and showed an example of this operation.

Definition 2.6 ([6]). We say that a binary operation $\cdot$ on $\mathcal{F}$ is product if it satisfies the following conditions:

(i) $\left(1_{\Omega}, 0_{\Omega}\right) \cdot\left(a_{1}, a_{2}\right)=\left(a_{1}, a_{2}\right)$ for each $\left(a_{1}, a_{2}\right) \in \mathcal{F}$;

(ii) the operation - is commutative and associative;

(iii) if $\left(a_{1}, a_{2}\right) \odot\left(b_{1}, b_{2}\right)=\left(0_{\Omega}, 1_{\Omega}\right)$ and $\left(a_{1}, a_{2}\right),\left(b_{1}, b_{2}\right) \in \mathcal{F}$, then

$$
\left(c_{1}, c_{2}\right) \cdot\left(\left(a_{1}, a_{2}\right) \oplus\left(b_{1}, b_{2}\right)\right)=\left(\left(c_{1}, c_{2}\right) \cdot\left(a_{1}, a_{2}\right)\right) \oplus\left(\left(c_{1}, c_{2}\right) \cdot\left(b_{1}, b_{2}\right)\right)
$$

and

$$
\left(\left(c_{1}, c_{2}\right) \cdot\left(a_{1}, a_{2}\right)\right) \odot\left(\left(c_{1}, c_{2}\right) \cdot\left(b_{1}, b_{2}\right)\right)=\left(0_{\Omega}, 1_{\Omega}\right)
$$

for each $\left(c_{1}, c_{2}\right) \in \mathcal{F}$;

(iv) if $\left(a_{1 n}, a_{2 n}\right) \searrow\left(0_{\Omega}, 1_{\Omega}\right),\left(b_{1 n}, b_{2 n}\right) \searrow\left(0_{\Omega}, 1_{\Omega}\right)$ and $\left(a_{1 n}, a_{2 n}\right),\left(b_{1 n}, b_{2 n}\right) \in \mathcal{F}$, then $\left(a_{1 n}, a_{2 n}\right) \cdot\left(b_{1 n}, b_{2 n}\right) \searrow\left(0_{\Omega}, 1_{\Omega}\right)$.

The following theorem gives an example of product operation for IF-events.

Theorem 2.7 ([6]). The operation · defined by

$$
\left(x_{1}, y_{1}\right) \cdot\left(x_{2}, y_{2}\right)=\left(x_{1} \cdot x_{2}, y_{1}+y_{2}-y_{1} \cdot y_{2}\right)
$$

for each $\left(x_{1}, y_{1}\right),\left(x_{2}, y_{2}\right) \in \mathcal{F}$ is product operation on $\mathcal{F}$.

Since now $\mathbf{m}_{x}: \mathcal{B}(R) \rightarrow[0,1]$ plays an analogous role as $P_{\xi}: \mathcal{B}(R) \rightarrow[0,1]$, we can define IF-mean value $\mathbf{E}(x)$ by the same formula (see [5]).

Definition 2.8 ([5]). We say that an IF-observable $x$ is an integrable IF-observable, if the integral $\int_{R} t d \mathbf{m}_{x}(t)$ exists. In this case we define IF-mean value

$$
\mathbf{E}(x)=\int_{R} t d \mathbf{m}_{x}(t) .
$$

If the integral $\int_{R} t^{2} d \mathbf{m}_{x}(t)$ exists, then we define IF-dispersion $\mathbf{D}^{2}(x)$ by the formula

$$
\mathbf{D}^{2}(x)=\int_{R} t^{2} d \mathbf{m}_{x}(t)-(\mathbf{E}(x))^{2}=\int_{R}(t-\mathbf{E}(x))^{2} d \mathbf{m}_{x}(t) .
$$

In [9] B. Riečan defined the notion of a joint IF-observable as follows:

Definition 2.9 ([9]). Let $x, y: \mathcal{B}(R) \rightarrow \mathcal{F}$ be two IF-observables. The joint IF-observable of the IF-observables $x$, y is a mapping $h: \mathcal{B}\left(R^{2}\right) \rightarrow \mathcal{F}$ satisfying the following conditions:

(i) $h\left(R^{2}\right)=\left(1_{\Omega}, 0_{\Omega}\right), h(\emptyset)=\left(0_{\Omega}, 1_{\Omega}\right)$; 
(ii) if $A, B \in \mathcal{B}\left(R^{2}\right)$ and $A \cap B=\emptyset$, then $h(A \cup B)=h(A) \oplus h(B)$ and $h(A) \odot h(B)=$ $\left(0_{\Omega}, 1_{\Omega}\right)$;

(iii) if $A, A_{1}, \ldots \in \mathcal{B}\left(R^{2}\right)$ and $A_{n} \nearrow A$, then $h\left(A_{n}\right) \nearrow h(A)$;

(iv) $h(C \times D)=x(C) \cdot y(D)$ for each $C, D \in \mathcal{B}(R)$.

Remark 2.10. The joint IF-observable of IF-observables $x$, y from Definition 2.9 is two-dimensional IF-observable.

Theorem 2.11 ([9]). For each two IF-observables $x, y: \mathcal{B}(R) \rightarrow \mathcal{F}$ there exists their joint IF-observable.

\section{Conditional IF-mean value for IF-observables}

In this section we present the notions connected with a conditional IF-mean value introduced in [4].

Definition 3.1 ([4]). If $x, y: \mathcal{B}(R) \rightarrow \mathcal{F}$ are the IF-observable and $B \in \mathcal{B}(R)$ is fixed, then we define $y_{x(B)}: \mathcal{B}(R) \rightarrow \mathcal{F}$ by the formula

$$
y_{x(B)}(D)= \begin{cases}\left(0_{\Omega}, 1_{\Omega}\right), & \text { if } D=\emptyset \\ y(D \backslash\{0\}) \cdot x(B), & \text { if } D \neq R, D \in \mathcal{B}(R) \\ \left(1_{\Omega}, 0_{\Omega}\right), & \text { if } D=R\end{cases}
$$

Proposition 3.1 ([4]). The mapping $y_{x(B)}$ is an IF-observable. If the IF-observable $y$ is integrable, then the IF-observable $y_{x(B)}$ is integrable, too.

In Definition 2.8 we have defined an integral $\int_{R} y d \mathbf{m}=\mathbf{E}(y)=\int_{R} t d \mathbf{m}_{y}(t)$, but in a conditional IF-mean value we need to work with the notion of an indefinite integral.

Definition 3.2 ([4]). If $x, y: \mathcal{B}(R) \rightarrow R$ are the IF-observables, such that $y$ is integrable, (i.e. there exists $\left.\mathbf{E}(y)=\int_{R} t d \mathbf{m}_{y}(t)\right)$, then the indefinite integral is defined by the formula

$$
\mathbf{E}\left(y_{x(B)}\right)=\int_{R} t d \mathbf{m}_{y_{x(B)}}(t)
$$

for fixed $B \in \mathcal{B}(R)$.

The next theorem says about an existence of a conditional IF-mean value.

Theorem 3.3 ([4]). Let $x, y: \mathcal{B}(R) \rightarrow R$ are the IF-observables, $y$ be integrable, i.e. there exists $\mathbf{E}(y)=\int_{R} t d \mathbf{m}_{y}(t)$. Then there exists a Borel measurable function $f: R \rightarrow R$ such that

$$
\mathbf{E}\left(y_{x(B)}\right)=\int_{B} f d \mathbf{m}_{x}
$$

for each $B \in \mathcal{B}(R)$. 
Now we are able to define a notion of conditional intuitionistic fuzzy mean value (expectation).

Definition 3.4 ([4]). If $x, y: \mathcal{B}(R) \rightarrow R$ are the IF-observables, $y$ is integrable, then the conditional IF-mean value (expectation) $\mathbf{E}(y \mid x)=f$ is the Borel measurable function such that

$$
\mathbf{E}\left(y_{x(B)}\right)=\int_{B} \mathbf{E}(y \mid x) d \mathbf{m}_{x}
$$

for each $B \in \mathcal{B}(R)$.

There is a connection between a conditional IF-mean value $\mathbf{E}(y \mid x)$ and a conditional IFprobability $\mathbf{p}(\mathbf{A} \mid x)$, see Remark 3.5. Recall that a conditional intuitionistic fuzzy probability is a Borel measurable function $f$ (i.e. $B \in \mathcal{B}(R) \Longrightarrow f^{-1}(B) \in \mathcal{B}(R)$ ) such that

$$
\int_{B} \mathbf{p}(\mathbf{A} \mid x) d \mathbf{m}_{x}=\mathbf{m}(\mathbf{A} \cdot x(B))
$$

for each $B \in \mathcal{B}(R)$, where $\mathbf{m}: \mathcal{F} \rightarrow[0,1]$ is the intuitionistic fuzzy state, $\mathbf{A} \in \mathcal{F}$ is an intuitionistic fuzzy event and $x: \mathcal{B}(R) \rightarrow \mathcal{F}$ is an intuitionistic fuzzy observable, see [8].

Remark 3.5 ([4]). Take $\mathbf{A} \in \mathcal{F}$ and define the IF-observable $y_{\mathbf{A}}: \mathcal{B}(R) \rightarrow \mathcal{F}$ by

$$
y_{\mathbf{A}}(B)= \begin{cases}\left(0_{\Omega}, 1_{\Omega}\right), & \text { if } B=\emptyset \\ \mathbf{A}, & \text { if } B=\{1\} \\ y_{\mathbf{A}}(B \cap\{1\}), & \text { if } B \neq \emptyset, B \neq R, B \in \mathcal{B}(R) \\ \left(1_{\Omega}, 0_{\Omega}\right), & \text { if } B=R\end{cases}
$$

Then $\mathbf{E}\left(y_{\mathbf{A}} \mid x\right)=\mathbf{p}(\mathbf{A} \mid x)$ holds $\mathbf{m}_{x}$-almost everywhere.

\section{Martingale convergence theorem for conditional IF-mean value}

Basic motivation for our calculus of IF-observables was the calculus with random variables. The following theorem works in the opposite direction in some sense: the conditional IF-mean value (expectation) of IF-observables can be represented by the conditional mean value (expectation) of random variables.

Theorem 4.1. Let $x, y: \mathcal{B}(R) \rightarrow \mathcal{F}$ be the IF-observables, y be an integrable. Let $h: \mathcal{B}(R) \rightarrow \mathcal{F}$ be the joint IF-observable of $x, y$. Consider the probability space $\left(R^{2}, \mathcal{B}\left(R^{2}\right), P=\mathbf{m} \circ h\right)$ and the random variables $\xi, \eta$ defined by $\xi((u, v))=u, \eta((u, v))=v$. Let $P_{\eta}$ be the probability distribution of $\eta$, i.e. $P_{\eta}(B)=P\left(\eta^{-1}(B)\right)$. Then $P_{\eta}=\mathbf{m}_{x}$ and $\mathbf{E}(y \mid x)=E(\xi \mid \eta)$ holds $\mathbf{m}_{x}$ almost everywhere. 
Proof. By the definition we have

$$
\begin{aligned}
P_{\eta}(B) & =P\left(\eta^{-1}(B)\right)=\mathbf{m} \circ h\left(\eta^{-1}(B)\right)=\mathbf{m}(h(R \times B))=\mathbf{m}(y(R) \cdot x(B))= \\
& =\mathbf{m}\left(\left(1_{\Omega}, 0_{\Omega}\right) \cdot x(B)\right)=\mathbf{m}(x(B))=\mathbf{m}_{x}(B)
\end{aligned}
$$

and

$$
\begin{gathered}
\int_{\eta^{-1}(B)} \xi d P=\int_{R \times B} u d(\mathbf{m} \circ h)(u, v), \\
\mathbf{E}\left(y_{x(B)}\right)=\int_{R} u d \mathbf{m}_{y_{x(B)}}(u)=\int_{R} u d \mu(u),
\end{gathered}
$$

where $B \in \mathcal{B}(R)$ is fixed and

$$
\mu(D)=\mathbf{m}(y(D) \cdot x(B))=\mathbf{m} \circ h(D \times B) .
$$

Now we prove that

$$
\int_{R \times B} f(u) d(\mathbf{m} \circ h)(u, v)=\int_{R} f d \mu
$$

for each Borel function $f: R \rightarrow R$. Put first $f=\lambda_{C}$. Then

$$
\begin{aligned}
\int_{R \times B} \lambda_{C}(u) d(\mathbf{m} \circ h)(u, v) & =\int_{R \times R} \lambda_{R \times B}(u, v) \cdot \lambda_{C \times R}(u, v) d(\mathbf{m} \circ h)(u, v)= \\
& =\int_{R \times R} \lambda_{C \times B}(u, v) d(\mathbf{m} \circ h)(u, v)=\mathbf{m}(h(C \times B))= \\
& =\mathbf{m}(y(C) \cdot x(B))=\mu(C)=\int_{R} \lambda_{C} d \mu .
\end{aligned}
$$

Equality (1) can be proved now by the linearity and continuity of the integrals. Put now $f(u)=u$. Then using (1) we have

$$
\begin{aligned}
\int_{B} \mathbf{E}(y \mid x) d \mathbf{m}_{x} & =\mathbf{E}\left(y_{x(B)}\right)=\int_{R} u d \mathbf{m}_{y_{x(B)}}(u)=\int_{R} u d \mu(u)= \\
& =\int_{R \times B} u d(\mathbf{m} \circ h)(u, v)=\int_{\eta^{-1}(B)} \xi d P=\int_{B} E(\xi \mid \eta) d P_{\eta}= \\
& =\int_{B} E(\xi \mid \eta) d \mathbf{m}_{x}
\end{aligned}
$$

for each $B \in \mathcal{B}(R)$. Hence $\mathbf{E}(y \mid x)=E(\xi \mid \eta)$ holds $\mathbf{m}_{x}$ - almost everywhere.

Theorem 4.2. (Martingale convergence theorem) Let $g_{n}: R \rightarrow R$ be the Borel measurable functions, $g_{n}^{-1}(\mathcal{B}(R)) \subset g_{n+1}^{-1}(\mathcal{B}(R))(n=1,2, \ldots), \bigcup_{n=1}^{\infty} g_{n}^{-1}(\mathcal{B}(R))=\mathcal{B}(R)$. Let $x: \mathcal{B}(R) \rightarrow$ $\mathcal{F}$ be an IF-observable, $x_{n}=x \circ g_{n}^{-1}, \mathbf{m}_{x} \ll \mathbf{m}_{x_{n}}(n=1,2, \ldots)$. Let $y: \mathcal{B}(R) \rightarrow \mathcal{F}$ be an integrable IF-observable. Then the convergence

$$
\mathbf{E}\left(y \mid x_{n}\right) \rightarrow \mathbf{E}(y \mid x) \text { holds } \mathbf{m}_{x} \text { - almost everywhere. }
$$


Proof. Let $\left(R^{2}, \mathcal{B}\left(R^{2}\right), P=\mathbf{m} \circ h\right)$ be the same probability space as in Theorem 4.1. Let $\xi, \eta$ be the coordinate random variables. By Theorem 4.1 we have

$$
P_{\eta}=\mathbf{m}_{x}, E(\xi, \eta)=\mathbf{E}(y \mid x) .
$$

Put

$$
\eta_{n}=g_{n} \circ \eta, \mathcal{S}_{n}=\eta^{-1}\left(g_{n}^{-1}(\mathcal{B}(R))\right)=\eta_{n}^{-1}(\mathcal{B}(R))
$$

Then

$$
\mathcal{S}_{n} \subset \mathcal{S}_{n+1}(n=1,2, \ldots)
$$

and

$$
\bigcup_{n=1}^{\infty} \mathcal{S}_{n}=\bigcup_{n=1}^{\infty} \eta^{-1}\left(g_{n}^{-1}(\mathcal{B}(R))\right)=\eta^{-1}\left(\bigcup_{n=1}^{\infty} g_{n}^{-1}(\mathcal{B}(R))\right)=\eta^{-1}(\mathcal{B}(R))=\mathcal{S}_{0} .
$$

By the martingale convergence theorem (see [10]) we have that the convergence

$$
E\left(\xi \mid \eta_{n}\right) \rightarrow E(\xi \mid \eta)
$$

holds $P_{\eta}$ - almost everywhere. Moreover

$$
\begin{aligned}
P_{\eta_{n}}(B) & =P\left(\eta^{-1}\left(g_{n}^{-1}(\mathcal{B}(R))\right)\right)=\mathbf{m}\left(h\left(R \times g_{n}^{-1}(B)\right)\right)=\mathbf{m}\left(y(R) \cdot x\left(g_{n}^{-1}(B)\right)\right)= \\
& =\mathbf{m}\left(\left(1_{\Omega}, 0_{\Omega}\right) \cdot x\left(g_{n}^{-1}(B)\right)\right)=\mathbf{m}\left(x_{n}(B)\right)=\mathbf{m}_{x_{n}}(B) .
\end{aligned}
$$

Hence $P_{\eta_{n}}(B)=\mathbf{m}_{x_{n}}(B)$. Further using Theorem 4.1 we obtain

$$
\begin{aligned}
\int_{B} E(\xi \mid \eta) d P_{\eta_{n}} & =\int_{\eta_{n}^{-1}(B)} \xi d P=\int_{\eta^{-1}\left(g_{n}^{-1}(B)\right)} \xi d P=\int_{g_{n}^{-1}(B)} E(\xi \mid \eta) d P_{\eta}= \\
& =\int_{g_{n}^{-1}(B)} \mathbf{E}(y \mid x) d \mathbf{m}_{x}=\int_{x\left(g_{n}^{-1}(B)\right)} y d \mathbf{m}=\int_{x_{n}(B)} y d \mathbf{m}= \\
& =\int_{B} \mathbf{E}\left(y \mid x_{n}\right) d \mathbf{m}_{x_{n}} .
\end{aligned}
$$

Since $P_{\eta_{n}}=\mathbf{m}_{x_{n}}$, we have that the convergence

$$
E\left(\xi \mid \eta_{n}\right)=\mathbf{E}\left(y \mid x_{n}\right)
$$

holds $\mathbf{m}_{x_{n}}$ - almost everywhere.

Of course, $\mathbf{m}_{x} \ll \mathbf{m}_{x_{n}}$, therefore the convergence

$$
E\left(\xi \mid \eta_{n}\right)=\mathbf{E}\left(y \mid x_{n}\right)
$$

holds $\mathbf{m}_{x}$ - almost everywhere.

Finally, the convergence

$$
\mathbf{E}\left(y \mid x_{n}\right)=E\left(\xi \mid \eta_{n}\right) \rightarrow E(\xi \mid \eta)=\mathbf{E}(y \mid x)
$$

holds $\mathbf{m}_{x}$ - almost everywhere. 


\section{Conclusion}

The paper concerns probability theory on intuitionistic fuzzy sets. In the paper, we showed the connection between a conditional IF-mean value of IF-observables and a conditional mean value of random variables. We formulated the modification of the martingale convergence theorem for conditional IF-mean value. Since the IF-probability can be decomposed on two IF-states, these results can be applicable for conditional IF-mean value induced by IF-probability.

\section{Acknowledgements}

This research was funded by Mobility project BAS-SAS-21-01.

\section{References}

[1] Atanassov, K. T. (2016). Intuitionistic fuzzy sets. VII ITKR Session, Sofia, 20-23 June 1983 (Deposed in Centr. Sci.-Techn. Library of the Bulg. Acad. of Sci., 1697/84) (in Bulgarian). Repr. Int. J. Bioautomation, 20, S1-S6.

[2] Atanassov, K. T. (2012). On Intuitionistic Fuzzy Sets, Springer, Berlin.

[3] Atanassov, K. T. (1999). Intuitionistic Fuzzy Sets: Theory and Applications, Physica Verlag, New York.

[4] Čunderlíková, K. (2021). Conditional intuitionistic fuzzy mean value. Axioms, 10(2), Article No. 97.

[5] Čunderlíková, K. (2020). A note on mean value and dispersion of intuitionistic fuzzy events. Notes on Intuitionistic Fuzzy Sets, 26 (4), 1-8.

[6] Lendelová, K. (2006). Conditional IF-probability. Advances in Soft Computing: Soft Methods for Integrated Uncertainty Modelling, 37, Springer, Berlin, Heidelberg, 275-283.

[7] Lendelová, K., \& Riečan, B. (2004). Weak law of large numbers for IF-events. Current Issues in Data and Knowledge Engineering, Bernard De Baets et al. eds., EXIT, Warszawa, 309-314.

[8] Riečan, B. (2012). Analysis of fuzzy logic models. Intelligent systems (V. Koleshko ed.), INTECH, 219-244.

[9] Riečan, B. (2006). On the probability and random variables on IF events. Applied Artifical Intelligence, Proc. 7th FLINS Conf. Genova, D. Ruan et al. eds., 138-145.

[10] Riečan, B., \& Neubrunn, T. (1997). Integral, Measure, and Ordering, Kluwer Academic Publishers, Dordrecht and Ister Science, Bratislava.

[11] Zadeh, L.A. (1965). Fuzzy sets. Information and Control, 8, 338-358.

[12] Zadeh, L. A. (1968). Probability measures on fuzzy sets. Journal of Mathematical Analysis and Applications, 23, 421-427. 